\title{
Clinical and Bacteriological Profile of Male Patients with Urethral Discharge at Western Regional Hospital, Nepal
}

\author{
Anand Nepal ${ }^{1}$, Kapil Subedi ${ }^{2}$, Jitendra Shah ${ }^{3}$, Manoj Koirala ${ }^{4}$ \\ ${ }^{1}$ Department of Dermatology, Pokhara Academy of Health Sciences, Western Regional Hospital, Pokhara, Nepal \\ ${ }^{2}$ Department of Dermatology, Green Pasteur Hospital, Pokhara, Nepal \\ ${ }^{3}$ Department of Microbiology, Pokhara Academy of Health Sciences, Pokhara, Nepal \\ ${ }^{4}$ Department of Medicine, Pokhara Academy of Health Sciences, Pokhara, Nepal
}

\section{Correspondence:}

Dr Anand Nepal, MD

Department of Dermatology, Venereology and Leprology, Pokhara Academy of Health Sciences, Western Regional Hospital, Pokhara, Nepal

Email: anandnpl@gmail.com.

Article accepted : $2^{\text {nd }}$ October, 2019
Article received : $28^{\text {th }}$ August, 2019

\section{ABSTRACT}

Background: Urethral discharge is a symptom of urethritis. Common organisms causing urethral discharge are Neisseria gonorrhea and Chlamydia trachomatis. Cephalosporines, Macrolides and Tetracycline groups of drugs are commonly used for the treatment.

Materials and Methods: This was a prospective, cross sectional, observational study. The patients were enrolled from the Sexually Transmitted Infections clinic and also from the hospital laboratory in between the period of one year. All the male cases with urethral discharge at STI clinic in between this period were included for clinical examination and laboratory testing. All urine and discharge specimens of urethral discharge patients as referred from other departments directly to the hospital laboratory for relevant tests were also included for the study.

Results: Neisseria gonorrhea was the most common agent of urethral discharge in male patients. Urethral discharge, dysuria and inguinal lymphadenopathy were common symptoms. All the Neisseria gonorrhea isolates were sensitive to Ceftriaxone and Imipenam.

Conclusion: Continuous monitoring with culture sensitivity testing of urethral discharge cases is required.

Key words: Ceftriaxone, drug resistance, gonorrhoea, Sexually Transmitted Infection, urethritis.

\section{INTRODUCTION}

Urethritis characterized by inflammation of urethra is a common presentation of Sexually Transmitted Infections (STIs). Symptoms of urethritis include discharge per urethra, pain during micturition, and frequent urination. ${ }^{1}$ Neisseria gonorrhoea (NG) and Chlamydia trachomatis (CT) are common etiological agents. Mycoplasma genitalium, Trichomonas vaginalis, Herpes simplex virus and adenoviruses are other causes. ${ }^{2}$

Ceftriaxone $250 \mathrm{mg}$ Intramuscular injection plus Azithromycin 1 gram per oral is recommended by Centre of Disease Control (CDC) for the treatment of uncomplicated gonococcal urethritis. ${ }^{3}$

World Health Organization (WHO) guidelines for the treatment of gonococcal urethritis recommend that local resistance data should determine the choice of therapy. 
In settings where local resistance data are not available, dual therapy of Ceftriaxone $250 \mathrm{mg}$ intramuscular (IM) as a single dose plus Azithromycin 1 gm orally as a single dose or Cefixime $400 \mathrm{mg}$ orally as a single dose plus azithromycin 1 gm orally as a single dose is recommended. ${ }^{4}$

Diagnosis of gonococcal infections can be made by visualization of intracellular gram negative diplococci in gram stained smear of the urethral discharge specimen. This technique is quick, inexpensive, and as sensitive as urethral specimen culture. ${ }^{5}$

The diagnosis of chlamydial infection is difficult. Direct cytological examination after Giemsa staining is relatively insensitive for genital tract infections. Isolation of the organism in cell cultures, antigen detection using enzyme immunoassays are few methods ${ }^{6}$. Other tests used for the diagnosis of urogenital CT infections include Nucleic Acid Amplification Tests (NAATs), direct immunofluorescence, and nucleic acid hybridization tests. $^{7}$

This was a prospective cross sectional observational study. The aim of the study was to observe the clinical findings and laboratory investigation results of male patients having urethral discharge together with sensitivity patterns of the organisms isolated from the urethral discharge specimens.

\section{MATERIALS AND METHODS}

All the male patients presenting to the Sexually Transmitted Infections (STI) clinic at Western Regional Hospital in between the period of 1st Jan 2018 to 1st Jan 2019 with complain of discharge per urethra were included. Data regarding the last sexual exposure pattern, precaution taken during the last sexual exposure, duration since onset of symptoms, clinical examination findings and treatments taken before presentation were collected.

Besides those from the STI clinic, specimens from male patients with urethral discharge referred from other departments to the laboratory for either microscopic examination or culture and sensitivity tests or both of the urine and/or urethral discharge specimen were also included.
The urethral exudates were collected from the urethral meatus by sterile cotton swabs. The specimens were smeared in a glass slide for gram stain and it was planted directly onto a split plate containing chocolate agar for culture sensitivity testing. After 24-48 hours of incubation were sub cultured for biochemical tests.

\section{RESULTS}

Total of 44 cases were enrolled from the STI clinic ranging from 15 to 56 years age with mean age of 29.43 years. Duration at presentation to us since the last sexual activity was minimum one day and maximum 240 days with mean duration 17.73 days. Twenty seven (61.4\%) patients were married and $17(38.6 \%)$ patients were unmarried. The last sexual activity was with the spouse in $17(38.6 \%)$ and extramarital in $27(61.4 \%)$ patients. Among the 44 patients, only two (4.5\%) had taken precaution by wearing condom and 42 (95.5\%) didn't wear condom during the last exposure. Urethral discharge was present since minimum one day and maximum 90 (mean 12.05) days and dysuria since minimum one and maximum 60 (mean 10.47) days before presenting to us. Dysuria was present in 36 patients $(81.8 \%)$ and inguinal lymphadenopathy in six $(13.6 \%)$ patients. Seven $(15.9 \%)$ had taken some kind of treatment from other clinic/ medical shops and 37(87.4\%) had not taken any treatment before presenting to us.

All the 44 cases presenting in the STI clinic were tested for Human Immunodeficiency Virus (HIV) serology and Venereal Disease Research Laboratory (VDRL) test. HIV was negative in all of the cases and (VDRL) test was positive in one $(2.3 \%)$ case.

Urine microscopic examination was done in 50 samples. Twenty (40\%) had urine pus cells less than five/ High Power Field (HPF) and $30(60 \%)$ had pus cells more than or equal to five $/ \mathrm{HPF}$.

Urethral exudate microscopic examinations for pus cells /HPF was done for 24 specimens which showed negative result (pus cells less than five/HPF) in 2(8.3\%) and positive (pus cells more than or equal to five/HPF) in $22(91.6 \%)$ cases. 
Gram staining was done for 49 specimens. Seven (14.28\%) specimens revealed gram positive cocci, $37(75.51 \%)$ intracellular gram negative diplococci and five $(10.25 \%)$ were negative.

Total of 52 samples were cultured. There were 47 samples with isolation of at least one bacteria. The growth patterns were Neisseria gonorrhea in 34 (72.3\%), Neisseria gonorrhea + Staph aureus in one $(2.12 \%)$, Staph aureus in eight(17.02\%), Staph epidermidis in three $(6.3 \%)$ and $\mathrm{E}$ coli in one $(2.12 \%)$ among the total isolates.

Figure 1: Growth pattern of microorganisms in chocolate agar media

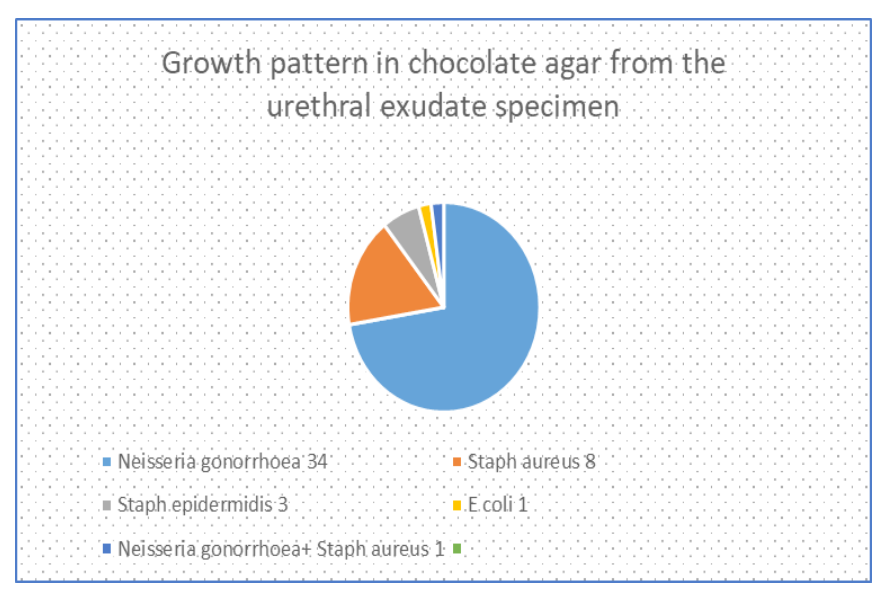

All the isolates tested to Imipenam $(\mathrm{n}=23)$ were sensitive to this drug, among which 17 were NG, five were Staph aureas, one was E coli and one of the Staph aureus isolate was Methicillin Resistant (MRSA).

Total of 42 isolates ( $\mathrm{n}=42)$ were tested for sensitivity to Ceftriaxone among which 33 were NG isolates, eight Staph aureus including the MRSA and one was E coli. All the isolates were sensitive to Ceftriaxone. Two of the NG isolates were only intermediately sensitive to Ceftriaxone.

Total of 26 isolates were tested for Cefixime $(n=26)$. Thirteen NG were sensitive but one NG isolate was only of intermediate sensitivity to Cefixime. E coli was sensitive to cefixime. Two of the Staph aureus were sensitive. The MRSA and nine NG isolates were resistant to Cefixime.

Total of 44 isolates were tested for sensitivity to
Azithromycin $(n=44)$ among which 21 NG, six Staph aureus including the MRSA and one E coli were resistant to Azithromycin. Fourteen NG isolates and two Staph aureus were sensitive to Azithromycin.

Total of 32 isolates were tested for Cotrimoxazole $(n=32)$ among which four Staph aureus including the MRSA, one E coli and $15 \mathrm{NG}$ were resistant. Nine NG and three Staph aureus were sensitive.

Total of 19 isolates were tested for Levofloxacin $(n=19)$ among which nine NG, one Staph aureus and one E coli were resistant. Six NG, one Staph epidermidis and one Staph aureus were sensitive.

Total of 23 isolates were tested for sensitivity to Ciprofloxacin $(n=23)$ among which seven $N G$ and six Staph aureus including the MRSA were resistant. Nine NG were sensitive and one staph aureus was of intermediate sensitivity.

Amikacin was tested in 26 isolates $(\mathrm{n}=26)$. Fifteen $\mathrm{NG}$, five Staph aureus including one MRSA and one Staph epidermidis were sensitive. Four NG and one Staph aureus were resistant.

\section{Table 1: Sensitivity of Neisseria gonorrhoea to different antibiotics.}

\begin{tabular}{|l|l|l|l|l|}
\hline Drug & $\begin{array}{l}\text { No. of } \\
\text { samples }\end{array}$ & $\begin{array}{l}\text { Sensi- } \\
\text { tive }\end{array}$ & $\begin{array}{l}\text { Resis- } \\
\text { tant }\end{array}$ & $\begin{array}{l}\text { Sensitive } \\
\text { Percentage }\end{array}$ \\
\hline Ceftriaxone & 33 & 33 & 0 & $100 \%$ \\
\hline Cefixime & 22 & 13 & 9 & $59.09 \%$ \\
\hline Azithromycin & 35 & 14 & 21 & $40 \%$ \\
\hline Cotrimoxazole & 24 & 9 & 15 & $37.50 \%$ \\
\hline Levofloxacin & 15 & 6 & 9 & $37.50 \%$ \\
\hline Imipenam & 17 & 17 & 0 & $100 \%$ \\
\hline Ciprofloxacin & 16 & 9 & 7 & $37.50 \%$ \\
\hline Amikacin & 19 & 15 & 4 & $37.50 \%$ \\
\hline
\end{tabular}

\section{DISCUSSION}

The mean age was 29.43 years for men in our study which is similar to other study done by Nayek AK et al. ${ }^{8}$ Gonococcal as well as chlamydial urethrits can be infected from symptomatic as well as asymptomatic infected individuals through sexual contact. ${ }^{9}$ In our study, most of the patients presented 
Original Article

late to the hospital even after the onset of symptoms (mean duration since onset of discharge was 12.05 days and dysuria was 10.47 days). Most of the patients $(61.4 \%)$ were married and in most of the cases $(61.4 \%)$ the last sexual exposure was not with the spouse. Together with these findings, 95.5\% of the patients in our study didn't wear a condom during their last sexual exposure. These findings point towards the likelihood that a patient of urethral discharge may pass the infection to their spouse or other sex partners before presenting to the hospital for the definitive treatment. The late presentation to the hospital in our settings may be because of less awareness and lack of knowledge of the condition or less availability of health centers.

There were no cases with co infections of HIV. Co infections with Syphilis was found to be very low among our patients compared to other studies. ${ }^{10}$

Microscopic examination after gram staining of 49 specimens of urethral discharge revealed seven(14.28\%) gram positive cocci, 37(75.51\%) intracellular gram negative diplococci and five $(10.25 \%)$ were negative. The finding of gram negative diplococci in these smears correlate with the findings in culture reports where $\mathrm{NG}$ were grown in $74.4 \%$. Thus it can be interpreted that the gram staining and microscopic evaluation of the urethral discharge is as good as culture testing in detecting NG infections. This finding is consistent with finding of Johnson RE et al and Lama L et al. ${ }^{11}$, 12

Chlamydial infections or co-infection of chlamydia together with other bacteria could not be detected in our study because we didn't do any investigation for detecting CT. The high percentage (8.3\%) of negative results in culture testing and high percentage (25.44\% among the total isolates) of isolation of bacterias other than NG may be because of not detecting mixed infection of $\mathrm{CT}$ with these bacterias.

Ceftriaxone resistant NG isolate has been found in various surveillence. ${ }^{13,14}$ In our study $32 \mathrm{NG}$ isolates were sensitive to ceftriaxone. But two of
Clinical and Bacteriological Profile of Male Patients. Nepal A et al. the NG isolates were only intermediately sensitive to ceftriaxone. These findings are similar to the study by Buder S et al in 2014 where among all the NG isolates sensitive to ceftriaxone, two NG isolates showed minimum inhibitory concentration (MIC) at estimated breakpoint $0.125 \mathrm{mg} / \mathrm{L} .{ }^{15}$ A study done from five cities of India by Kulkarni SV et al (2018) and also another study by T Wi et al found decreased susceptibility of NG isolates to ceftriaxone similar to our findings. ${ }^{16,17}$ In our study one $\mathrm{NG}(4.76 \%)$ isolate was only of intermediate sensitivity to cefixime. Nine NG isolates (42.85\%) were resistant to cefixime. The resistance of NG to cefixime in our study was much higher than that of Buder S et al (2014, 2015), Lee RS et al, Kulkarni SV et al, and Lama L et al but close to the Global surveillance report 2017 for south east asia. ${ }^{12,15-18}$

\section{CONCLUSION}

NG is the predominant cause of urethritis. Public education systems in our country should increase the health education programmes to spread knowledge about sexually transmitted infections and their preventive measures among the sexually active populations.

Sensitivity of NG to Ceftriaxone is decreasing. Resistance of NG to Cefixime, Azithromycin, Cotrimoxazole, Levofloxacin and Ciprofloxacin are established. Imipenam can be considered as an alternative treatment in NG urethritis. Further large scale studies are needed to follow up our findings.

\section{REFERENCES}

1. Vigneswaran HT, Baird G, Hwang K, Renzulli $\mathrm{J}$, Chan PA. Etiology of symptomatic urethritis in men and association with sexual behaviors. R I Med J 2013. 2016 Jun 1;99(6):37-40.

2. Bachmann LH, Manhart LE, Martin DH, Seña AC, Dimitrakoff J, Jensen JS, et al. Advances in the Understanding and Treatment of Male Urethritis. Clin Infect Dis. 2015 Dec 15;61(suppl_8):S763-9.

3. Gonococcal Infections - 2015 STD Treatment Guidelines [Internet]. 2018 [cited 2018 Sep 18]. 
Available from: https://www.cdc.gov/std/tg2015/ gonorrhea.htm

4. World Health Organization, Reproductive Health and Research. WHO guidelines for the treatment of Neisseria gonorrhoeae. [Internet]. 2016 [cited 2018 Sep 18]. Available from: http:// www.ncbi.nlm.nih.gov/books/NBK379221/

5. Judson FN. Gonococcal Urethritis-Diagnosis and Treatment. Arch Androl. 1979 Jan; 3(4):32935 .

6. Chernesky MA. The laboratory diagnosis of Chlamydia trachomatis infections. Can J Infect Dis Med Microbiol. 2005;16(1):39-44.

7. Keegan MB, Diedrich JT, Peipert JF. Chlamydia trachomatis Infection: Screening and Management. J Clin Outcomes Manag JCOM. 2014 Jan;21(1):308.

8. Nayak AK, Anoop TV, Sacchidanand S. A clinico-etiological study of urethritis in men attending sexually transmitted disease clinic at a tertiary hospital. Indian J Sex Transm Dis AIDS. 2017;38(2):136-41.

9. Hill SA, Masters TL, Wachter J. Gonorrhea an evolving disease of the new millennium. Microb Cell. 2016;3(9):371-389. Published 2016 Sep 5. doi:10.15698/mic2016.09.524.

10. Gonorrhoea \& its co-infection with other ulcerative, non-ulcerative sexually transmitted \& HIV infection in a Regional STD Centre [Internet]. [cited 2019 Aug 27]. Available from: https://www. ncbi.nlm.nih.gov/pmc/articles/PMC3103165/

11. Johnson RE, Newhall WJ, Papp JR, Knapp JS, Black CM, Gift TL, et al. Screening tests to detect Chlamydia trachomatis and Neisseria gonorrhoeae infections--2002. MMWR Recomm Rep Morb Mortal Wkly Rep Recomm Rep. 2002 Oct 18;51(RR-15):1-38; quiz CE1-4.

12. Lama L, Kayestha BMM. Gonococcal culture and drug sensitivity pattern in patients with urethral discharge and vaginal discharge with cervicitis. Post-Grad Med J NAMS [Internet]. 2017 Sep 7 [cited 2018 Aug 14];13(2). Available from: https:// pmjn.org.np/index.php/pmjn/article/view/222

13. Epidemiological Trends of Antibiotic Resistant Gonorrhoea in the United Kingdom [Internet]. [cited 2019 Aug 27]. Available from: https://www. ncbi.nlm.nih.gov/pmc/articles/PMC6165062/

14. Ohnishi M, Golparian D, Shimuta K, Saika T, Hoshina S, Iwasaku K, et al. Is Neisseria gonorrhoeae Initiating a Future Era of Untreatable Gonorrhea?: Detailed Characterization of the First Strain with High-Level Resistance to Ceftriaxone. Antimicrob Agents Chemother. 2011 Jul;55(7):3538-45.

15. Buder S, Dudareva S, Jansen K, Loenenbach A, Nikisins S, Sailer A, et al. Antimicrobial resistance of Neisseria gonorrhoeae in Germany: low levels of cephalosporin resistance, but high azithromycin resistance. BMC Infect Dis. 2018 17;18(1):44.

16. Lee RS, Seemann T, Heffernan H, Kwong JC, Gonçalves da Silva A, Carter GP, et al. Genomic epidemiology and antimicrobial resistance of Neisseria gonorrhoeae in New Zealand. J Antimicrob Chemother. 2018 Feb 1;73(2):353-64.

17. Kulkarni SV, Bala M, Muqeeth SA, Sasikala G, Nirmalkar AP, Thorat R, et al. Antibiotic susceptibility pattern of Neisseria gonorrhoeae strains isolated from five cities in India during 2013-2016. J Med Microbiol. 2018 Jan;67(1):22-8.

18. Wi T, Lahra MM, Ndowa F, Bala M, Dillon J-AR, Ramon-Pardo P, et al. Antimicrobial resistance in Neisseria gonorrhoeae: Global surveillance and a call for international collaborative action. PLoS Med [Internet]. 2017 Jul 7 [cited 2019 Aug 22];14(7). Available from: https://www.ncbi.nlm. nih.gov/pmc/articles/PMC5501266/ 